\title{
Sönke Wortmann setzt sich für Implantatgesundheit ein
}

Auf einer gemeinsamen Pressekonferenz zur Aktion gesundes Zahnimplantat informierten Sönke Wortmann (Regisseur, Produzent und Patient der Kaiserberg Klinik), Dr. Richard Meissen (Ärztlicher Direktor und Leiter der Implantologie/Oralchirurgie der Kaiserberg Klinik), Sylvia Fresmann (Vorsitzende der Deutschen Gesellschaft für Dentalhygieniker/-innen e.V.) und Jan-Philipp Schmidt (Geschäftsführer des Aktionsbündnisses gesundes Implantat) am 13. September in der Kaiserberg Klinik in Duisburg über die Vorbeugung von parodontalen Erkrankungen und die Notwendigkeit der sorgfältigen Pflege von dentalen Implantaten.

Um die Patientenmotivation in diesem Bereich weiter zu stärken, finalisiert das Aktionsbündnis gesundes Implantat aktuell gemeinsam mit den Entwicklern Tobias und Philip Reisberger (VentureMedica/RKSB) eine ImplantatPass-App. Die App enthält als digitale und erweiterte Variante des beim Bündnis stark nachgefragten ImplantatPasses für Sicherheit \& Pflege neben einer Terminfunktion zur Implantatprophylaxe in der Praxis und Informationen zu den eigenen Implantaten, Hinweise zur Implantatpflege, ein Ärzteverzeichnis mit Suchfunktion, individuelle Produktempfehlungen und viele weitere Features.

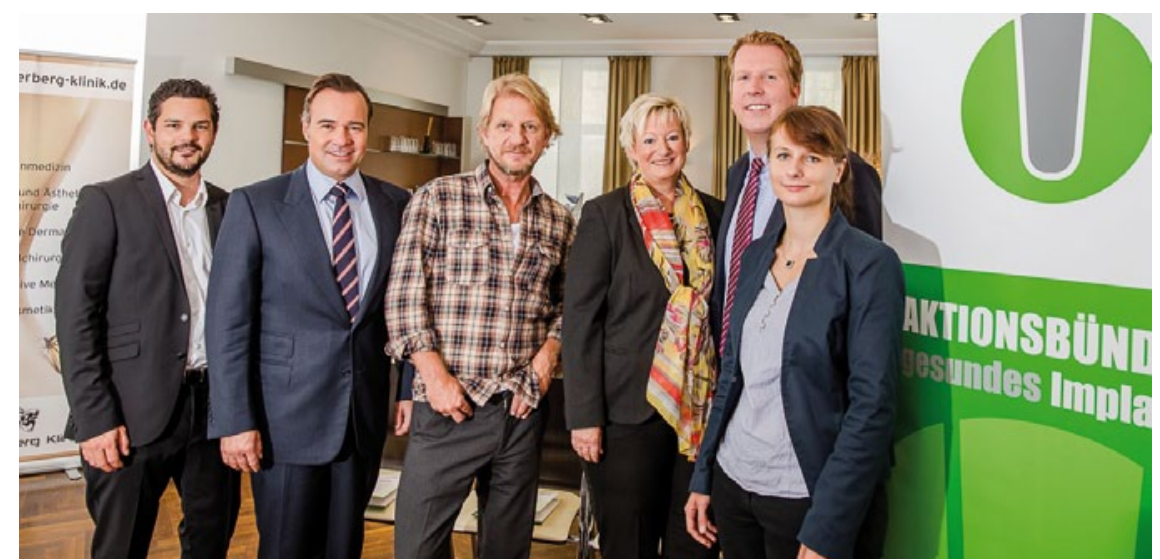

V.I.n.r.: P. Reisberger, R. Meissen, S. Wortmann, S. Fresmann, J.-P. Schmidt und K. Jahn (Projektmanagerin Aktionsbündnis gesundes Implantat).

„Es ist uns wichtig, dass sich Patienten entsprechend ihrer persönlichen Vorlieben zwischen dem digitalen und dem gedruckten Pass entscheiden können - nur ein ImplantatPass, der auch wirklich genutzt wird, kann zur Prävention periimplantärer Erkrankungen beitragen“, erklärt Jan-Philipp Schmidt, Geschäftsführer des Aktionsbündnisses gesundes Implantat.

Im Rahmen der Aktion gesundes Zahnimplantat können sich Patienten in der Kaiserberg Klinik mit dem Stichwort „Aktionsbündnis gesundes Implantat“ bis zum 31. Oktober 2013 kostenfrei auf Entzündungen mit Gewebeabbau an Zahnim- plantaten testen lassen. Unter der Telefonnummer 0203-39360 kann man sich hierfür unkompliziert anmelden. Unterstützt wird das Projekt vom Aktionsbündnis gesundes Implantat, das sich als bislang einmaliger Zusammenschluss von Unternehmen, Verbänden, Fachexperten und Fachverlagen für Prophylaxe bei Zahnimplantaten einsetzt. Die App zum ImplantatPass für Sicherheit und Pflege wird voraussichtlich im November 2013 verfügbar sein.

Nach einer Pressemitteilung des Aktionsbündnis gesundes Implantat, Leipzig Internet: www.gesundes-implantat.de

\section{Dynamischer Mittelståndler feiert 25-Jahre-Jubilåum}

Die curasan AG feiert in diesen Tagen ihr 25-jähriges Bestehen. In einer Feierstunde mit der Geschäftsleitung gratulierten Vertreter der Gemeinde Kleinostheim und der IHK Aschaffenburg dem seit über 2 Jahrzehnten in der Region ansässigen Spezialisten für Knochenregeneration. Der 1. Bürgermeister Kleinostheims, Hubert Kammerlander, beglückwünschte das Unternehmen zu seiner Erfolgsgeschichte und brachte seine Anerkennung zum Ausdruck: „Wir sind sehr stolz darauf, dass wir eine so innovative Firma hier bei uns in Kleinostheim haben." Herr Andreas Elsner, Geschäftsbereich Innovation und Umwelt der IHK Aschaffenburg, schloss sich den Glückwünschen an und überreichte dem Vorstandsvorsitzenden Hans Dieter
Rössler die Ehrenurkunde der IHK zum 25-jährigen Bestehen.

Hans Dieter Rössler hat das Unternehmen mit Weitblick gesteuert. Mit sicherem Gespür für neue Strömungen und Entwicklungen im Gesundheitswesen verwandelte er die einstige Pharmafirma, die mit Plasmaderivaten, generischen Antibiotika und Anästhetika handelte, in einen Spezialisten für Medizinprodukte im Bereich der regenerativen Knochenchirurgie. Das mittlerweile zu einer Dachmarke ausgebaute synthetische Knochenaufbaumaterial CERASORB ${ }^{\circledR}$ gilt als offizieller weltweiter Referenz-
Standard für diese Materialklasse und zählt zu den wissenschaftlich am besten dokumentierten Produkten.

Zum Einsatz kommen die Produkte in der Knochenund Geweberegeneration, Gewebezüchtung, Wundheilung sowie bei der Arthrosebehandlung. Sie richten sich an Orthopäden, Unfall- und Wirbelsäulenchirurgen, Sportmediziner, Implantologen, Mund-, Kiefer und Gesichtschirurgen sowie andere chirurgisch tätige Zahnärzte.

Nach einer Pressemitteilung der curasan AG, Kleinostheim

Internet: www.curasan.de 


\title{
Das ITI Scholarship-Programm als Karrieresprungbrett
}

Pro Jahr vergibt das ITI (International Team for Implantology) im Rahmen seines Scholarship-Programms 20 Stipendien und unterstützt sowohl die Stipendiaten als auch die Scholarship-Zentren finanziell.

Nach Aussage des ITI verfolge man mit dem Scholarship-Programm das Ziel, die Fortbildung junger Kliniker auf dem Gebiet der dentalen Implantologie, den internationalen Austausch sowie die fachliche Vernetzung zu fördern. Mittels eines Stipendiums von 30075,-€ ermöglicht das ITI jungen Zahnärzten, 12 Monate an einem der weltweit 20 ITI-ScholarshipZentren zu verbringen und ihr Wissen in der dentalen Implantologie zu vertiefen. Die ITI-Scholarship-Zentren sind renommierte Fachabteilungen an Kliniken oder Universitäten.

Die Organisation griff seit 1998 weit mehr als 200 graduierten Nachwuchs-

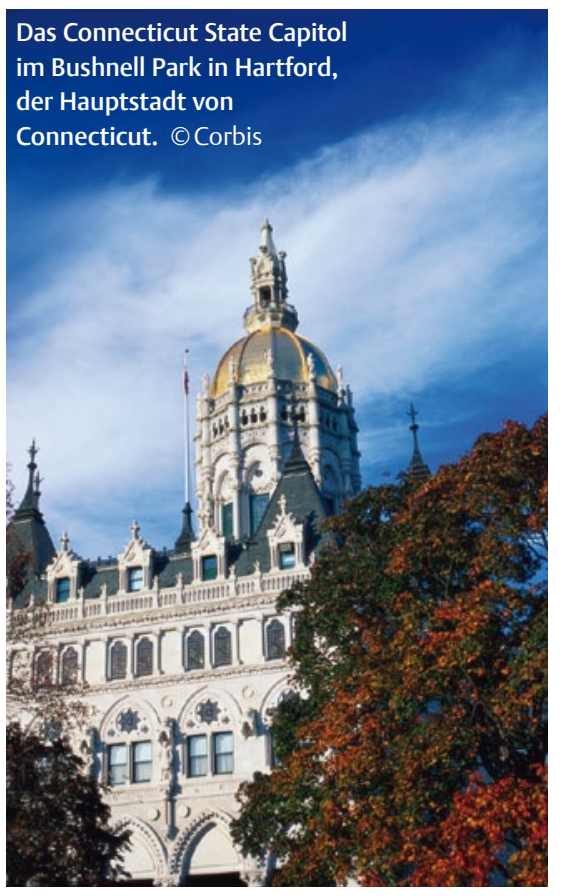

zahnärzten aus aller Welt finanziell unter die Arme. Das für die Evaluierung und die Auswahl zuständige ITI-Scholarship-Komitee verzeichnete in den vergangenen Jahren eine kontinuierlich wachsende Anzahl an Bewerbungen für das Programm. Die aktuell 20 Scholarship-Zentren können dabei vom Antragsteller nach eigenen Behandlungs- und Fortbildungsschwerpunkten frei gewählt werden.

Zahlreiche Informationen und Hintergrundtexte zum ITI-Scholarship-Programm finden sich auf der Internetseite des ITI (www.iti.org). Die Bewerbungsfrist läuft jährlich vom 1. Juni bis zum 8. Juli. Bei einer Bewerbung sollte man berücksichtigen, dass der Auswertungsprozess seitens des Prüfungskomitees mehrere Monate dauern kann.

Nach einer Pressemitteilung der ITI Sektion Deutschland, Freiburg Internet: www.iti.org/germany

\section{Divisionen und Deutschland-Vertrieb neu aufgestellt \\ Marktanforderungen im Blick}

\begin{abstract}
Heraeus Kulzer hat seine Strukturen auf veränderte Marktanforderungen ausgerichtet. Der klare Fokus liegt auf den Bereichen „Dental Materials" und „Digital Servi-
\end{abstract} ces". Vor diesem Hintergrund hat

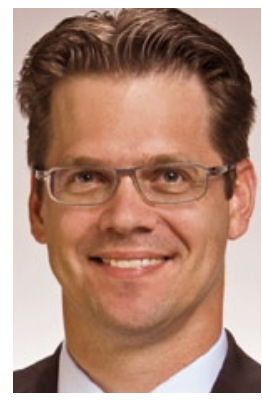

A. Bacher

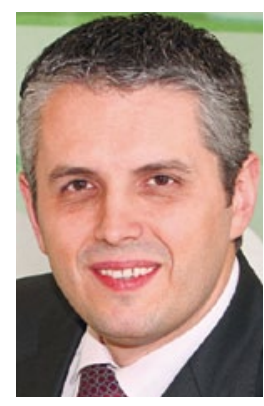

N. Savic

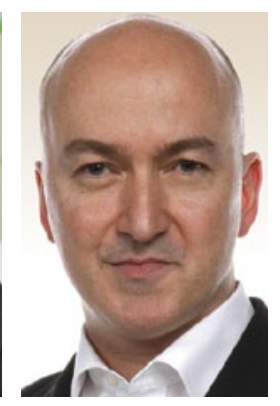

N. Weber

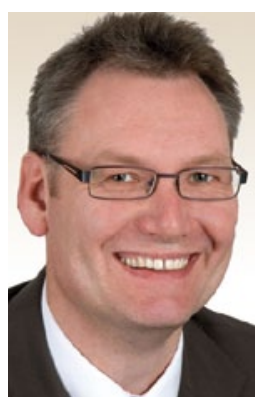

J. Bewersdorf

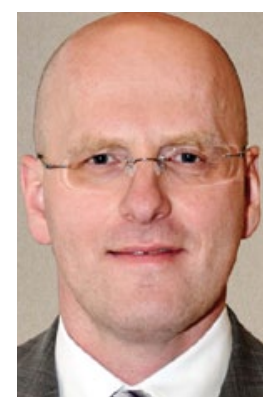

J. Scheffler das Unternehmen auch den Vertrieb in Die Division entwickelt und vermarktet Deutschland neu aufgestellt.

Dr. Andreas Bacher leitet seit Mitte Mai die neue Division Digital Services. Spezialisten aus Informationstechnologie, Zahnmedizin und -technik arbeiten dort Hand in Hand, um die Anwender im Praxis- und Laboralltag im digitalen cara-Workflow zu unterstützen. Bacher ist seit 2007 für Heraeus tätig, zuletzt als Leiter der Business Unit Packaging Technology bei der Heraeus Materials Technology GmbH \& Ko. KG. Der 41-Jährige war bis 2011 Leiter Corporate Development bei der Heraeus Holding. Novica Savic leitet seit Jahresbeginn den Geschäftsbereich der Dental Materials.
Werkstoffe für Zahnärzte und Zahntechniker. Künftig arbeiten die Experten für Praxis- und Labormaterialien noch enger zusammen. Savic war von 1996 bis 2008 für Heraeus Kulzer tätig. Nach einem kurzen Gastspiel bei Nobel Biocare leitete der 43-Jährige ab 2010 die Division Dentistry der Hanauer. Norbert Weber verantwortet seit Jahresbeginn das internationale Produktmanagement der Dentalmaterialien. Jens Bewersdorff ist für die internationale Marketingkommunikation der Division verantwortlich.

Als Country Manager Deutschland verantwortet Jörg Scheffler seit Jahresbeginn die Vertriebs- und Marketing-Aktivitäten für den Zahnarzt- und Labormarkt. Als Mitglied des globalen Managements von Heraeus Kulzer und Vice-President berichtet Scheffler direkt an Geschäftsführer Dr. Martin Haase. Der 46-Jährige ist seit 2007 für Heraeus Kulzer tätig, zuletzt als Country Manager Prothetik \& Digital Deutschland, Österreich und Schweiz. Bianca Laubach unterstützt das Marketing in Deutschland. Neben dem Kurs- und Eventmanagement ist ihr Team für die Planung und Umsetzung marktspezifischer Maßnahmen zuständig.

Nach einer Pressemitteilung der Heraeus Kulzer GmbH, Hanau 